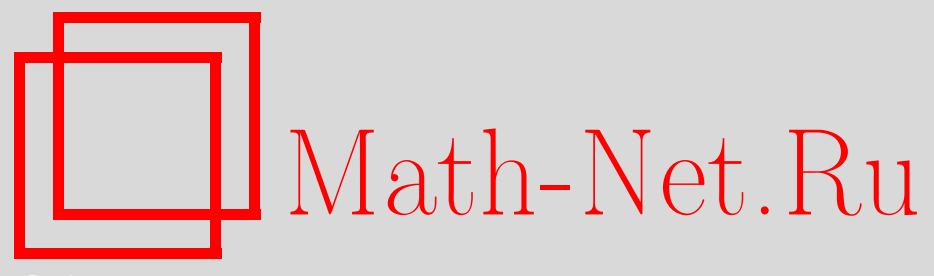

С. М. Дудаков, Монадические состояния над упорядоченным универсальным случайным графом и конечные автоматы, Изв. РАН. Сер. матем., 2011, том 75, выпуск $5,47-64$

DOI: https://doi.org/10.4213/im4077

Использование Общероссийского математического портала Math-Net.Ru подразумевает, что вы прочитали и согласны с пользовательским соглашением http://www . mathnet.ru/rus/agreement

Параметры загрузки:

IP: 54.237 .59 .107

26 апреля 2023 г., 12:54:53

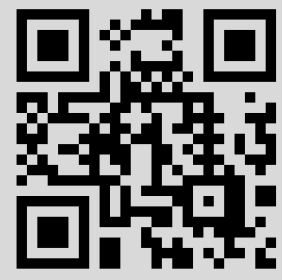




\title{
С. М. Дудаков \\ Монадические состояния над упорядоченным универсальным случайным графом и конечные автоматы
}

\begin{abstract}
Продолжено изучение выразительной силы языка логики предикатов для конечных алгебраических систем, вложенных в бесконечные, которое было начато в работах М. А. Тайцлина, М. Бенедикта, Л. Либкина и др. Изучено, какие свойства конечной монадической системы можно выразить с помощью формул, если вложить такую систему в линейно упорядоченный произвольным образом случайный граф. Использовано представление Бюхи для связи монадических состояний и формальных языков. Показано, что если ограничиться рассмотрением только <-инвариантных в линейно упорядоченных случайных графах формул, то такие формулы соответствуют конечным автоматам. Продемонстрировано, что =-инвариантные формулы, выражающие собственные свойства вложенных систем, способны выразить только булеву комбинацию свойств вида “мощность пересечения одноместных предикатов принадлежит одной из конечного числа фиксированных конечных или бесконечных арифметических прогрессий”.

Библиография: 18 наименований.
\end{abstract}

Ключевые слова: универсальный случайный граф, логика первого порядка, автоматный язык.

\section{§1. Введение}

Языки математической логики и их выразительная сила для конечных алгебраических систем в последнее время активно исследуются, поскольку они находят широкое применение на практике, например в системах управления базами данных (см. [1], [2]), математической моделью которых являются конечные алгебраические системы. Хорошо известно, что не все свойства конечных алгебраических систем могут быть выражены с помощью формул логики первого порядка. В частности, таким простейшим невыразимым свойством является определение четности числа элементов одноместного предиката (см. [3]).

Одним из способов увеличения выразительной силы языка первого порядка является вложение конечной алгебраической системы в некоторую бесконечную систему (универсум) и использование в логических формулах отношений вкладываемой конечной системы и отношений универсума (см. [4]). Для некоторых универсумов такой способ действительно расширяет выразительные возможности языка. Например, Ю. Ш. Гуревичем доказано (но не опубликовано; см. [5]), что наличие в универсуме линейного порядка расширяет класс выразимых свойств. Интересно, что во многих случаях дальнейшее увеличение

Работа выполнена при финансовой поддержке РФФИ (гранты № 10-01-00532, 08-01-00241). 
сигнатуры универсума не приводит к росту выразительных возможностей языка логики предикатов (см. [6]-[9] и обзор в [5]).

Тем не менее, существуют линейно упорядоченные универсумы, для которых происходит дальнейшее увеличение выразительной силы. Так, в [10], [11] показано, что если конечная алгебраическая система $\mathfrak{D}$ вложена в линейно упорядоченный (универсальный) случайный граф $(\mathfrak{G},<)$, то в системе $(\mathfrak{G},<, \mathfrak{D})$ можно выразить четность количества элементов любого одноместного предиката из $\mathfrak{D}$. Примечательно, что по отдельности как линейный порядок, так и случайный граф этого не позволяют (см. [3] и [12] соответственно). Другой результат получен в [13]: при вложении системы $\mathfrak{D}$ в неупорядоченный случайный граф выразительная сила языка логики первого порядка возрастает до монадической логики второго порядка над $\mathfrak{D}$.

Возникает естественный вопрос: какие вообще свойства системы $\mathfrak{D}$ могут быть выражены с помощью формул первого порядка, если система $\mathfrak{D}$ вложена в линейно упорядоченный случайный граф $(\mathfrak{G},<)$ ?

В одном частном случае, когда случайный граф упорядочен по типу натуральных чисел, ответ получен в [10]. Такая система может рассматриваться как модель теории наследственно конечных множеств, следовательно, в данном универсуме можно выразить любое арифметическое, в частности рекурсивное или рекурсивно-перечислимое, свойство.

Однако существуют и другие линейные упорядочения случайного графа даже с разрешимой теорией (см. [14]). Поэтому представляет интерес, в том числе и практический, следующий вопрос: какие свойства вкладываемых конечных систем можно выразить с помощью формул над линейно упорядоченным случайным графом независимо от способа упорядочения?

В настоящей работе мы полностью исследуем этот вопрос в случае, когда сигнатура $\Omega$ системы $\mathfrak{D}$ является монадической, т. е. система $\mathfrak{D}$ состоит только из одноместных предикатов.

Используя алгебраическое представление Бюхи, мы доказываем, что с помощью <-инвариантных в линейно упорядоченных случайных графах формул можно выразить только такие свойства, которые соответствуют автоматным языкам. Далее мы рассматриваем =-инвариантные формулы, выражающие собственные свойства системы $\mathfrak{D}$, и доказываем, что такие формулы способны выразить только булеву комбинацию свойств следующего вида: мощность пересечения $R_{1}^{*} \cap \cdots \cap R_{n}^{*}$ равна одному из $N_{1}, \ldots, N_{m}$ или имеет вид $k j+l$ для некоторого натурального $j$. Здесь $m, k, l, N_{1}, \ldots, N_{m}$ - натуральные константы, а через $R_{i}^{*}$ мы обозначили $R_{i}$ или его дополнение.

\section{§ 2. Определения}

Графом называется алгебраическая система с двухместным предикатом $E$. Элементы графа традиционно называются вершинами. Говорят, что граф не имеет петель, если $E(x, x)$ ложно для любой вершины $x$.

ОПРеДЕЛЕНИЕ 1 (см. также [15]). Счетный граф $\mathfrak{G}=(V, E)$ называется случайным, если для любых четырех конечных множеств вершин $A, B, C, D$, для которых $A \cap B=C \cap D=\varnothing$, существует вершина $x$ такая, что: 
1) $(x, y) \in E$ для любой вершины $y \in A$;

2) $(x, y) \notin E$ для любой вершины $y \in B$;

3) $(y, x) \in E$ для любой вершины $y \in C$;

4) $(y, x) \notin E$ для любой вершины $y \in D$.

Здесь мы дали вариант определения для ориентированных графов. Оригинально в [15] дано аналогичное определение для неориентированных графов, т. е. в случае, когда отношение $E$ симметрично.

В работе [14] показано, что существует разрешимое линейное упорядочение случайного графа, точнее, теория такой системы допускает эффективную элиминацию кванторов.

ТеОРема 1 [14]. На случайном графе без петель $\mathfrak{G}$ существует линейный порядок < такой, что в теории $\mathrm{Th}(\mathfrak{G},<)$ формула

$$
\begin{aligned}
(\exists x)\left((a<x<b) \wedge\left(\bigwedge_{i} E\left(c_{i}, x\right)\right)\right. & \wedge\left(\bigwedge_{i} \neg E\left(d_{i}, x\right)\right) \\
& \left.\wedge\left(\bigwedge_{i} E\left(x, e_{i}\right)\right) \wedge\left(\bigwedge_{i} \neg E\left(x, f_{i}\right)\right)\right)
\end{aligned}
$$

эквивалентна формуле

$$
a<b \wedge\left(\bigwedge_{i \neq j} c_{i} \neq d_{j}\right) \wedge\left(\bigwedge_{i \neq j} e_{i} \neq f_{j}\right) .
$$

Иначе говоря, на каждом непустом интервале существуют вершины, связанные с заданными вершинами указанным образом.

Зафиксируем некоторую конечную предикатную сигнатуру $\Omega$, которую, следуя традиции, будем называть сигнатурой базы данных. Алгебраические системы сигнатуры $\Omega$ будем называть состояниями базы данных, или просто $\Omega$-состояниями. Через $|\mathfrak{B}|$ будем, как обычно, обозначать носитель алгебраической системы $\mathfrak{B}$.

ОПРЕДЕЛЕНИЕ 2 [4]. Пусть $\Omega$ - сигнатура базы данных. Пусть $\mathfrak{A}$ - некоторая алгебраическая система сигнатуры $\Sigma$. Если $\mathfrak{D}-\Omega$-состояние, для которого $|\mathfrak{D}| \subseteq|\mathfrak{A}|$, то $\mathfrak{D}$ называется $\Omega$-состоянием над $\mathfrak{A}$. В этом случае будем называть $\mathfrak{A}$ универсумом, а $\Sigma$ - сигнатурой универсума. Активной областью $\Omega$-состояния $\mathfrak{D}$ над $\mathfrak{A}$ (обозначаем $\operatorname{adom}(\mathfrak{D})$, или просто adom, если понятно, какое именно $\Omega$-состояние имеется в виду) назовем множество элементов $\mathfrak{A}$, участвующих хотя бы в одном отношении из $\mathfrak{D}$.

Заметим, что при переходе от системы $\mathfrak{D}=\left(D, R_{1}, \ldots, R_{n}\right)$ к системе $\left(\mathfrak{A}, R_{1}, \ldots, R_{n}\right)$ теряется информация о количестве элементов системы $\mathfrak{D}$, не входящих ни в одно из отношений $\mathfrak{D}$. Для того чтобы этой потери не происходило, мы в дальнейшем считаем, что активная область $\mathfrak{D}$ совпадает с носителем $\mathfrak{D}: \operatorname{adom}(\mathfrak{D})=|\mathfrak{D}|$. Также отметим, что множество $\operatorname{adom}(\mathfrak{D})$ определимо с помощью $\Omega$-формулы ввиду конечности сигнатуры $\Omega$.

Если сигнатура $\Sigma$ не имеет общих символов с $\Omega$, то можно рассматривать единую алгебраическую систему $(\mathfrak{A}, \mathfrak{D})$ и формулы сигнатуры $(\Sigma, \Omega)$. 
ОПРЕДЕЛЕНИЕ 3. Пусть $\Omega$ - сигнатура базы данных. Пусть $K$ - класс систем сигнатуры $\Sigma$. Формула $\phi$ сигнатуры $(\Sigma, \Omega)$ выражает свойство $S \Omega$-состояний над универсумами из $K$, если для любого $\Omega$-состояния $\mathfrak{D}$ над любым универсумом $\mathfrak{A}$ из $K$ выполняется следующее: $(\mathfrak{A}, \mathfrak{D}) \models \phi$ тогда и только тогда, когда $(\mathfrak{A}, \mathfrak{D})$ обладает свойством $S$.

Нас будут интересовать прежде всего те свойства и формулы, которые зависят от самого состояния, но не зависят от способа вложения $\mathfrak{D}$ в $\mathfrak{A}$.

ОПРЕДЕЛЕНИЕ 4 [16]. Пусть $\Omega$ - сигнатура базы данных. Пусть $\mathfrak{A}_{1}$ и $\mathfrak{A}_{2}-$ алгебраические системы сигнатуры $\Sigma$. Пусть $\Sigma_{0} \subseteq \Sigma$. Пусть $f$ - изоморфизм между $\Omega$-состоянием $\mathfrak{D}_{1}$ над универсумом $\mathfrak{A}_{1}$ и $\Omega$-состоянием $\mathfrak{D}_{2}$ над универсумом $\mathfrak{A}_{2}$. Говорим, что $f$ - это $\Sigma_{0}$-изоморфизм $\mathfrak{D}_{1}$ и $\mathfrak{D}_{2}$, если $f$ сохраняет все отношения из $\Sigma_{0}$ в обе стороны.

Пусть $K$ - класс систем сигнатуры $\Sigma$. Свойство $S$ (и формула $\phi$, его выражающая) называется $\Sigma_{0}$-инвариантным в $K$, если оно сохраняется при произвольных $\Sigma_{0}$-изоморфизмах произвольного $\Omega$-состояния $\mathfrak{D}$ над универсумами из $K$.

В качестве сигнатуры универсума мы всюду будем использовать сигнатуру упорядоченного случайного графа $(E,<)$, а класс $K$ будет состоять из всевозможных линейно упорядоченных случайных графов. Если $\Sigma_{0}=\varnothing$, то $\Sigma_{0}$-инвариантные свойства и формулы называем $=-$ инвариантными, если $\Sigma_{0}=(<)$, то -<-инвариантными. Таким образом, =-инвариантные свойства это "собственные" свойства $\Omega$-состояний, вообще не связанные с универсумом, a <-инвариантные свойства зависят только от упорядочения элементов $\Omega$-состояния.

Сведения о конечных автоматах можно найти, например, в [17]. Конечнъм автоматом называем пятерку $\left(Q, \Sigma, P, q_{0}, F\right)$, в которой $Q$ - конечное множество состояний автомата, $\Sigma$ - входной алфавит, $q_{0} \in Q$ - начальное состояние, $F \subseteq Q-$ множество принимающих состояний, $P$ - множество команд вида

$$
q a \rightarrow p_{1} \vee \cdots \vee p_{n},
$$

где $a \in \Sigma, q, p_{1}, \ldots, p_{n} \in Q$. Если $n=1$ для всех команд из $P$, то автомат называется детерминированным. Автомат принимает слово $w=w_{1} \ldots w_{m}$, где $w_{1}, \ldots, w_{m} \in \Sigma$, если существует последовательность состояний $q_{0}, q_{1}, \ldots, q_{m} \in Q$ такая, что в $P$ есть команды вида

$$
q_{i-1} w_{i} \rightarrow \cdots \vee q_{i} \vee \cdots
$$

для всех $i=1, \ldots, m$, при этом $q_{m} \in F$.

Если $\Sigma$ - алфавит, то $\Sigma$-языком называется множество конечных слов алфавита $\Sigma$. С помощью $\otimes$ будем обозначать операцию "посимвольного соединения" для слов одинаковой длины. Точнее, если $x=x_{1} \ldots x_{n}-$ слово в алфавите $\Sigma_{1}$, а $y=y_{1} \ldots y_{n}-$ слово в алфавите $\Sigma_{2}$, то с помощью $x \otimes y$ обозначаем следующее слово в алфавите $\Sigma_{1} \times \Sigma_{2}$ :

$$
\left(x_{1}, y_{1}\right) \ldots\left(x_{n}, y_{n}\right) .
$$

Для слов разной длины считаем $x \otimes y$ неопределенным. 
Если $L-\Sigma_{1}$-язык, то $\Sigma_{2}$-цилиндрификациями языка $L$ называем языки $\left\{w \otimes u: w \in L, u \in \Sigma_{2}^{*}\right\}$ и $\left\{u \otimes w: w \in L, u \in \Sigma_{2}^{*}\right\}$, где $\Sigma_{2}^{*}-$ множество всех слов алфавита $\Sigma_{2}$.

Пусть все буквы алфавита $\Sigma$ имеют вид $\left(a_{1}, \ldots, a_{n}\right)$. Пусть буквы алфавита $\Sigma^{\prime}$ получены из букв алфавита $\Sigma$ удалением $i$-й компоненты. Будем говорить, что $\Sigma^{\prime}$-слово $w^{\prime}$ является $i$-проекиией $\Sigma$-слова $w$, если $w^{\prime}$ может быть получено из $w$ вычеркиванием $i$-й компоненты в каждой из букв, $i$-проекиией $\Sigma$-языка $L$ называется $\Sigma^{\prime}$-язык $L^{\prime}$, состоящий из $i$-проекций всех слов из $L$.

Множество слов, принимаемых конечным автоматом $\mathfrak{M}$, называется языком, распознаваемым автоматом $\mathfrak{M}$. Язык, распознаваемый некоторым конечным автоматом, называется автоматным. Общеизвестны следующие теоремы (см., например, [17]).

Теорема 2. Объединение и дополнение автоматных языков, а также иилиндрификация и проекиия автоматного языка снова являются автоматньми языками.

ТЕОрема 3. Каждый автоматный язык распознается некоторым детерминированным конечным автоматом.

Сигнатура $\Omega$, состоящая только из одноместных предикатных символов, называется монадической. Состояния сигнатуры $\Omega$ будем в этом случае тоже называть монадическими.

Как показано в [12], изучая монадические состояния, всегда можно предполагать, что одноместные предикаты не пересекаются. В самом деле, пусть сигнатура базы данных $\Omega^{\prime}$ состоит из $n^{\prime}$ унарных предикатных символов: $\Omega^{\prime}=\left(P_{1}, \ldots, P_{n^{\prime}}\right)$. Тогда в любом $\Omega^{\prime}$-состоянии эти предикаты разбивают носитель этого состояния в общем случае на $2^{n^{\prime}}$ непересекающихся частей. Введем новые одноместные предикатные символы $R_{i}, i=0, \ldots, 2^{n^{\prime}}-1$, для обозначения этих частей. Теперь можем считать, что вместо монадической сигнатуры базы данных $\Omega^{\prime}$ мы оперируем с монадической сигнатурой $\Omega=\left(R_{0}, R_{1}, \ldots, R_{n}\right)$, $n=2^{n^{\prime}}-1$, такой, что предикаты, обозначаемые через $R_{i}$, не пересекаются. Предикат $R_{0}$, соответствующий ложности всех $P_{1}, \ldots, P_{n^{\prime}}$, как мы указали при определении активной области состояний, всегда пуст, поэтому не нужен. Любая формула сигнатуры $\left(\Sigma, \Omega^{\prime}\right)$ эффективно транслируется в формулу сигнатуры $(\Sigma, \Omega)$ : достаточно каждую атомную формулу вида $P_{i}(x)$ заменить на дизъюнкцию формул вида $\bigvee_{R_{j} \subseteq P_{i}} R_{j}(x)$. Обратное преобразование так же легко осуществимо: если есть формула сигнатуры $\Omega$, то нужно заменить каждую атомную формулу вида $R_{j}(x)$ на соответствующую ему конъюнкцию формул вида $P_{i}(x)$ и $\neg P_{i}(x)$.

Далее мы рассматриваем только сигнатуру базы данных $\Omega$, которая состоит из одноместных предикатных символов $R_{1}, \ldots, R_{n}$, попарно не имеющих общих элементов.

C каждым конечным $\Omega$-состоянием $\mathfrak{D}$ над произвольным упорядоченным универсумом $\mathfrak{A}$ можно связать некоторое слово $\mathfrak{d}(\mathfrak{D})$, его описывающее, следующим образом. 
ОПРЕДЕЛЕНИЕ 5 (представление Бюхи). Пусть $\Omega=\left(R_{1}, \ldots, R_{n}\right)$ - сигнатура базы данных. Пусть $\mathfrak{A}$ - линейно упорядоченный универсум. Пусть $N-$ мощность активной области $\Omega$-состояния $\mathfrak{D}, y_{1}<\cdots<y_{N}$ - все элементы активной области, упорядоченные по возрастанию. Для сигнатуры $\Omega$ определим алфавит $\widetilde{\Omega}=\left\{r_{1}, \ldots, r_{n}\right\}$. Состояние $\mathfrak{D}$ называется представлением Бюхи для $\widetilde{\Omega}$-слова $\mathfrak{d}(\mathfrak{D})=r_{j_{1}} \ldots r_{j_{N}}$, если для любого $i=1, \ldots, N$ выполнено $R_{j_{i}}\left(y_{i}\right)$. Слово $\mathfrak{d}(\mathfrak{D})$ будем называть прообразом Бюхи для $\mathfrak{D}$.

Пусть $S$ - некоторое <-инвариантное свойство состояний. Будем говорить, что $S$ задает в алгебраической системе $\mathfrak{A}$ следующий $\widetilde{\Omega}$-язык:

$$
L_{S}=\{\mathfrak{d}(\mathfrak{D}):(\mathfrak{A}, \mathfrak{D}) \text { обладает свойством } S\} .
$$

Аналогично, если $\phi-<$-инвариантная замкнутая формула сигнатуры $(\Sigma, \Omega)$, то ф задает $\widetilde{\Omega}$-язык в алгебраической системе $\mathfrak{A}$ :

$$
L_{\phi}=\{\mathfrak{d}(\mathfrak{D}):(\mathfrak{A}, \mathfrak{D}) \models \phi\} .
$$

Приведем следующий известный результат.

Теорема 4 (теорема Трахтенброта-Бюхи-Элгота). Совокупность автоматных языков совпадает с совокупностью языков, задаваемых монадическими формулами второго порядка на упорядоченных состояниях (без вложения в универсумы).

Далее мы будем рассматривать только линейно упорядоченный некоторым образом случайный граф $(\mathfrak{G},<)$, поэтому будем подразумевать, что язык задается именно в системе $(\mathfrak{G},<)$, и не будем упоминать об этом специально.

ОПРЕДЕЛЕНИЕ 6 . Пусть $(\mathfrak{G},<)$ - линейно упорядоченный случайный граф, $A \subseteq|\mathfrak{G}|-$ конечное множество, $y_{1}<\cdots<y_{N}$ - все элементы $A$, упорядоченные по возрастанию, $z \in|\mathfrak{G}|$. Через $\mathfrak{z}(z, A)$ обозначим слово в алфавите $\mathrm{E}=\{0,1,2,3\}$ длины $N$, в котором на $i$-м месте стоит одна из следующих цифр:

1) 0, если выполнено $\neg E\left(z, y_{i}\right)$ и $\neg E\left(y_{i}, z\right)$;

2) 1 , если выполнено $\neg E\left(z, y_{i}\right)$ и $E\left(y_{i}, z\right)$;

3) 2 , если выполнено $E\left(z, y_{i}\right)$ и $\neg E\left(y_{i}, z\right)$;

4) 3 , если выполнено $E\left(z, y_{i}\right)$ и $E\left(y_{i}, z\right)$.

Итак, для произвольного $\Omega$-состояния $\mathfrak{D}$ над упорядоченным случайным графом $(\mathfrak{G},<)$ определен прообраз Бюхи: $\widetilde{\Omega}$-слово $\mathfrak{d}(\mathfrak{D})$ длины, совпадающей с размером активной области. Кроме того, для любого элемента $z$ случайного графа будет определено $\mathfrak{z}(z, \operatorname{adom}(\mathfrak{D}))-$ Е-слово той же длины. В дальнейшем для краткости мы будем пропускать скобки с аргументами и записывать просто $\mathfrak{d}$ вместо $\mathfrak{d}(\mathfrak{D})$. Аналогично, вместо записи вида $\mathfrak{z}(u, \operatorname{adom}(\mathfrak{D}))$ мы будем просто использовать готический вариант буквы-аргумента: $\mathfrak{u}$.

\section{§ 3. Автоматные формулы и конечные автоматы}

Из работы [13] и теоремы Трахтенброта-Бюхи-Элгота следует, что каждый конечный автомат $\mathfrak{M}$ соответствует некоторой формуле $\phi_{\mathfrak{M}}$ сигнатуры $(E,<, \Omega)$, причем автомат принимает слово d тогда и только тогда, когда 
формула $\phi_{\mathfrak{M}}$ истинна для представления Бюхи слова $\mathfrak{d}$, вложенного в случайный граф. Нам потребуются некоторые дополнительные сигнатурные требования к таким формулам, поэтому мы дадим следующее определение.

Для удобства будем считать, что имеются две константы $m$ и $M$. Первая обозначает наименьший элемент активной области, вторая - наибольший. Также будем использовать одноместный функциональный символ $s$, определенный на всей активной области, кроме $M$, и обозначающий следующий по порядку элемент активной области. Терм $s(M)$ считаем неопределенным, а любую атомную формулу с его вхождением - ложной. Нетрудно видеть, что все эти символы определимы формулами в сигнатуре $(<, \Omega)$.

ОПредЕЛЕНИЕ 7. Формулу сигнатуры $(E,<, \Omega, m, M, s)$ назовем автоматной, если она имеет вид

$$
(\exists \bar{x} \notin \operatorname{adom})(\forall y \in \operatorname{adom}) \phi(\bar{x}, y, \bar{z}),
$$

где $\phi$ - булева комбинация формул вида $E(v, y), E(y, v), E(v, s(y)), E(s(y), v)$, $E(v, m), E(v, M), E(m, v), E(M, v), y=M, R_{i}(y)$; здесь $v$ - одна из переменных наборов $\bar{x}$ и $\bar{z}$.

Покажем, что выразительная сила языка таких формул остается достаточной для моделирования произвольного конечного автомата.

ТеОРема 5. Пусть $\Omega=\left(R_{1}, \ldots, R_{n}\right)$ - сигнатура базы данных. Для всякого натурального $k$ и конечного автомата $\mathfrak{M}$ с входным алфавитом $\mathrm{E}^{k} \times \widetilde{\Omega}$ можно эффективно построить автоматную формулу $\psi_{\mathfrak{M}}\left(z_{1}, \ldots, z_{k}\right)$ такую, что в любом линейно упорядоченном случайном графе $(\mathfrak{G},<)$ формула $\psi_{\mathfrak{M}}\left(z_{1}, \ldots\right.$ $\left.\ldots, z_{k}\right)$ истинна на $\Omega$-состоянии $\mathfrak{D}$ над $(\mathfrak{G},<)$ тогда и только тогда, когда автомат М воспринимает слово $\mathfrak{z}_{1} \otimes \cdots \otimes \mathfrak{z}_{k} \otimes \mathfrak{d}$.

ДокАзАТЕльство аналогично доказательствам похожих утверждений о моделировании работы конечных автоматов. Мы дадим способ построения этой формулы, а то, что она удовлетворяет нужным требованиям, легко проверяется по индукции.

Прежде всего, согласно теореме 3 мы можем считать, что $\mathfrak{M}$ - детерминированный автомат. Пусть автомат $\mathfrak{M}$ имеет вид $\left(Q, \mathrm{E}^{k} \times \widetilde{\Omega}, P, q_{0}, F\right)$, где программа $P$ является множеством команд вида

$$
q\left(\mathrm{z}_{1}, \ldots, \mathrm{z}_{k}, r\right) \rightarrow p
$$

$q, p \in Q, \mathrm{z}_{1}, \ldots, \mathrm{z}_{k} \in \mathrm{E}, r \in \widetilde{\Omega}$. Мы построим автоматную формулу

$$
(\exists \bar{x} \notin \operatorname{adom})(\forall y \in \operatorname{adom}) \phi(\bar{x}, y, \bar{z}),
$$

в которой длина набора $\bar{x}$ будет равна мощности $Q$, а формула $\phi$ будет конъюнкцией четырех формул: $\phi_{0}, \phi_{P}, \phi_{F}$ и $\phi_{B}$. Для удобства сразу пронумеруем элементы набора $\bar{x}$ элементами $Q:$ с помощью $x_{q}$ будем обозначать соответствующий $q$ элемент $\bar{x}$. Неформально говоря, истинность $E\left(x_{q}, y_{i}\right)$, где $y_{i}-i$-й по порядку элемент активной области, будет означать, что в $i$-й момент времени автомат находится в состоянии $q$. 
Формула $\phi_{0}$ будет описывать начальное состояние автомата: $\phi_{0} \equiv E\left(x_{q_{0}}, m\right)$.

Формула $\phi_{P}$ будет иметь вид $y \neq M \rightarrow \phi_{P}^{\prime}$, где $\phi_{P}^{\prime}-$ конъюнктивная нормальная форма (КНФ), описывающая работу автомата в соответствии с программой $P$. Для каждой команды $q\left(\mathrm{z}_{1}, \ldots, \mathrm{z}_{k}, r_{i}\right) \rightarrow p$ из $P$ мы включим в $\phi_{P}^{\prime}$ следующую элементарную дизъюнкцию:

$$
E\left(x_{q}, y\right) \wedge E^{*}\left(z_{1}, y\right) \wedge \cdots \wedge E^{*}\left(z_{k}, y\right) \wedge R_{i}(y) \rightarrow E\left(x_{p}, s(y)\right) .
$$

С помощью $E^{*}\left(z_{j}, y\right)$ мы обозначаем формулы следующего вида:

1) $\neg E\left(z_{j}, y\right) \wedge \neg E\left(y, z_{j}\right)$ при $\mathrm{z}_{j}=0$;

2) $\neg E\left(z_{j}, y\right) \wedge E\left(y, z_{j}\right)$ при $z_{j}=1$;

3) $E\left(z_{j}, y\right) \wedge \neg E\left(y, z_{j}\right)$ при $z_{j}=2$;

4) $E\left(z_{j}, y\right) \wedge E\left(y, z_{j}\right)$ при $z_{j}=3$.

Формула $\phi_{M}$ будет описывать состояние автомата, из которого автомат может перейти в принимающее состояние:

$$
\phi_{M} \equiv \bigvee_{\left[q\left(\mathrm{z}_{1}, \ldots, \mathrm{z}_{k}, r\right) \rightarrow p\right] \in P, p \in F} E\left(x_{q}, M\right) \wedge E^{*}\left(z_{1}, M\right) \wedge \cdots \wedge E^{*}\left(z_{k}, M\right) \wedge R(M) .
$$

Наконец, $\psi_{B}$ будет налагать ограничение: в каждый момент времени должно быть в точности одно состояние

$$
\psi_{B} \equiv \bigvee_{q \in Q}\left(E\left(x_{q}, y\right) \wedge \bigwedge_{p \neq q} \neg E\left(x_{p}, y\right)\right)
$$

Доказательство корректности построения стандартное.

Предположим, автомат $\mathfrak{M}$ принимает слово. Возьмем в качестве значений для $x_{q}$ такой элемент случайного графа, чтобы было $E\left(x_{q}, y_{i}\right)$ тогда и только тогда, когда автомат $\mathfrak{M}$ к $i$-му символу переходит в состоянии $q$. Тогда для любого $y \in$ adom все формулы будут истинны.

С другой стороны, если формула $\phi$ истинна, то нетрудно показать, что автомат $\mathfrak{M}$ принимает слово: в каждый момент времени $i$ он будет находиться в состоянии $q$, если выполнено $E\left(x_{q}, y_{i}\right)$. Поскольку истинна будет и формула $\phi_{M}$, на последнем шаге автомат перейдет в принимающее состояние.

Обращение следующей теоремы непосредственно вытекает из работы [13] и теоремы Трахтенброта-Бюхи-Элгота.

Теорема 6. Пусть $\Omega=\left(R_{1}, \ldots, R_{n}\right)$ - сигнатура базы данных. Для всякого натурального $k$ и всякой автоматной формуль $\theta\left(z_{1}, \ldots, z_{k}\right)$ можно эффективно построить конечный автомат $\mathfrak{M}_{\theta}$ со входным алфавитом $\mathrm{E}^{k} \times \widetilde{\Omega}$ такой, что в любом линейно упорядоченном случайном графе $(\mathfrak{G},<)$ автомат $\mathfrak{M}_{\theta}$ принимает слово $\mathfrak{z}_{1} \otimes \cdots \otimes \mathfrak{z}_{k} \otimes \mathfrak{d}$ тогда и толъко тогда, когда формула $\theta\left(z_{1}, \ldots, z_{k}\right)$ истинна на $\Omega$-состоянии $\mathfrak{D}$ над $(\mathfrak{G},<)$.

СледствиЕ 1. Любая формула, составленная из автоматных формул, эквивалентна автоматной.

ДокАЗАТЕЛЬСтво легко получается из теорем 5, 6. 
При построении формулы, как нетрудно заметить, отрицание и квантор существования будут соответствовать дополнению и проекции языков. Дизъюнкция двух формул соответствует цилиндрификации языков с последующим объединением.

Из теорем 5, 6 также легко получить несколько следствий.

СЛЕДСТВИЕ 2. Пусть $(\mathfrak{G},<)$ - произволъное линейное упорядочение случайного графа. Тогда класс языков, задаваемых автоматными формулами, совпадает с классом автоматных языков.

СлеДСТвИЕ 3. Любая замкнутая автоматная формула является <-инвариантной для линейно упорядоченных случайных графов.

\section{§ 4. <-инвариантные формулы}

Напомним, что мы рассматриваем конечные монадические состояния над линейно упорядоченными случайными графами.

Перед доказательством главной теоремы о <-инвариантных формулах над упорядоченными случайными графами установим несколько вспомогательных утверждений, справедливых для любого линейного упорядочения случайного графа.

Лемма 1. Существует автоматная формула $\Phi_{1}(x)$, которая для любого $\Omega$-состояния над линейно упорядоченным случайным графом утверждает следующее:

(*) существует в точности один элемент $y \in$ adom, для которого выполнено $E(x, y)$, и для всех $y \in$ adom не выполнено $E(y, x)$.

ДокАзАТЕЛЬСтво. Нетрудно видеть, что в терминах языков условие сводится к тому, что в Е-слове $\mathfrak{x}=\mathfrak{z}(x$, adom $)$ существует в точности одна единица, а остальные символы являются нулями. Это, очевидно, легко проверяется конечным автоматом, поэтому нужная формула существует по теореме 5.

Лемма 2. Пусть $x_{1}$ и $x_{2}$ удовлетворяют условию (*). Обозначим с помощъю $y_{1}$ и $y_{2}$ элементы из adom, для которых выполнены $E\left(x_{1}, y_{1}\right)$ и $E\left(x_{2}, y_{2}\right)$. Существуют автоматные формуль $\Phi_{=}\left(x_{1}, x_{2}\right), \Phi_{<}\left(x_{1}, x_{2}\right)$ u $\Phi_{s}\left(x_{1}, x_{2}\right)$, которые для любого $\Omega$-состояния над линейно упорядоченным случайным графом означают, что $y_{1}=y_{2}, y_{1}<y_{2}$ и $s\left(y_{1}\right)=y_{2}$ соответственно.

Доказательство. Напомним: условие $(*)$ для $x$ означает, что слово $\mathfrak{x}$ состоит из нулей, кроме места, соответствующего $y$, где стоит единица. Кроме того, слова $\mathfrak{x}_{1}$ и $\mathfrak{x}_{2}$ имеют одинаковую длину.

Таким образом, первая формула соответствует тому, что единицы в $\mathfrak{x}_{1}$ и $\mathfrak{x}_{2}$ стоят на одном и том же месте; вторая формула - единица в слове $\mathfrak{x}_{1}$ находится на следующей позиции по отношению к единице в слове $\mathfrak{x}_{2} ;$ третья - единица в $\mathfrak{x}_{2}$ стоит сразу после единицы в $\mathfrak{x}_{1}$. Легко построить конечные автоматы, проверяющие каждое из этих условий, поэтому нужные формулы существуют по теореме 5 . 
Лемма 3. Пусть $x$ удовлетворяют условию (*). Обозначим с помощъю у элемент из adom, для которого выполнено $E(x, y)$. Тогда существуют автоматные формуль $\Phi_{\min }(x)$ и $\Phi_{\max }(x)$, утверждающие, что у - соответственно наименьший и наибольший элемент из adom.

ДокАзАТЕльство аналогично доказательствам предыдущих лемм. Условия означают, что в слове $\mathfrak{x}$ единица стоит на первом или, соответственно, на последнем месте.

Лемма 4. Существует автоматная формула $\Phi_{e}$, утверждающая, что мошность аdom равна единице.

Доказательство. В качестве $\Phi_{e}$ берем $(\forall y \in$ adom $) y=M$.

Следующая лемма верна не для любого упорядочения случайного графа, а только для специального.

Лемма 5. Пусть случайный граф $\mathfrak{G}$ упорядочен способом из теоремы 1. Пусть $\phi(u, \bar{v}, \bar{w}, \bar{a}, \bar{b}, \bar{c}, \bar{d})$ - автоматная формула, а $\psi$ - элементарная конбюнкиия вида

$$
\begin{aligned}
\left(\bigwedge_{i} u<v_{i}\right) & \wedge\left(\bigwedge_{i} w_{i}<u\right) \wedge\left(\bigwedge_{i} E\left(u, a_{i}\right)\right) \\
& \wedge\left(\bigwedge_{i} \neg E\left(u, b_{i}\right)\right) \wedge\left(\bigwedge_{i} E\left(c_{i}, u\right)\right) \wedge\left(\bigwedge_{i} \neg E\left(d_{i}, u\right)\right) .
\end{aligned}
$$

Тогда формула $(\exists u)(\psi \wedge \phi)$ эквивалентна для состояний над $(\mathfrak{G},<)$ формуле

$$
(\exists u) \phi \wedge\left(\bigwedge_{i, j} w_{i}<v_{j}\right) \wedge\left(\bigwedge_{i, j} a_{i} \neq b_{j}\right) \wedge\left(\bigwedge_{i, j} c_{i} \neq d_{j}\right),
$$

если все переменные наборов $\bar{a}, \bar{b}, \bar{c}, \bar{d}$ не принадлежат adom.

ДокАЗАТЕЛЬСтво. Импликация в прямую сторону очевидна: для $u$ можно взять то же самое значение.

Предположим, выполнено (1) для некоторого $u$. Определим следующие конечные множества:

$$
\begin{array}{ll}
A=\left\{a_{i}\right\}_{i} \cup\{x \in \text { adom: } E(u, x)\}, & B=\left\{b_{i}\right\}_{i} \cup\{x \in \text { adom: } \neg E(u, x)\}, \\
C=\left\{c_{i}\right\}_{i} \cup\{x \in \text { adom: } E(x, u)\}, & D=\left\{d_{i}\right\}_{i} \cup\{x \in \text { adom: } \neg E(x, u)\} .
\end{array}
$$

Очевидно, $A \cap B=C \cap D=\varnothing$. Пусть $w$ - наибольшее из $w_{i}, v$ - наименьшее из $v_{i}$. Далее остается применить теорему 1 , чтобы получить $u^{\prime}$ такое, что $w<u^{\prime}<v$, $E\left(u^{\prime}, x\right)$ для $x \in A, \neg E\left(u^{\prime}, x\right)$ для $x \in B, E\left(x, u^{\prime}\right)$ для $x \in C, \neg E\left(x, u^{\prime}\right)$ для $x \in D$. Для этого $u^{\prime}$, очевидно, будет выполнена формула $\psi$. С другой стороны, в автоматную формулу ф будут входить только формулы $E(u, y)$ и $E(y, u)$ для $y \in$ adom, а значения таких формул для $u^{\prime}$ остались теми же, что и для $u$. Поэтому формула $\phi$ для $u^{\prime}$ также будет истинной.

Теперь докажем основное утверждение. 
ТеоремА 7. Любая замкнутая <-инвариантная над упорядоченными случайными графами формула $\mu$ сигнатуры $(E,<, \Omega)$ эквивалентна в них некоторой автоматной формуле $\lambda$, которую можно эффективно построить по $\mu$.

ДокАЗАтЕльство. Выберем упорядоченный граф из теоремы 1 и будем рассматривать только такие состояния над ним, что для любых $y_{1}, y_{2} \in$ adom выполнено $\neg E\left(y_{1}, y_{2}\right)$. Поскольку формула $\mu<$-инвариантна для линейно упорядоченных случайных графов, от такого изменения универсума и активной области значение $\mu$ измениться не должно.

Выполним несколько предварительных шагов по преобразованию формулы $\mu$ к нужному виду.

Шаг 1. Приведем формулу $\mu$ к предваренной форме, а затем разделим каждый квантор на два, по активной области и по ее дополнению:

$$
\begin{aligned}
&(\exists x) \phi \equiv(\exists x \in \text { adom }) \phi \vee(\exists x \notin \text { adom }) \phi, \\
&(\forall x) \phi \equiv(\forall x \in \text { adom }) \phi \wedge(\forall x \notin \text { adom }) \phi .
\end{aligned}
$$

Теперь для каждой переменной точно известно, принадлежит она активной области или не принадлежит. Заметим, что для любых $x_{1}, x_{2} \in$ adom будем иметь ложность $E\left(x_{1}, x_{2}\right)$, поэтому в дальнейшем считаем, что такие формулы отсутствуют. Аналогично, ложными формулами будут $R(u)$ для $u \notin$ adom, поэтому и такие формулы можно исключить.

Снова приведем $\mu$ к предваренной форме.

Шаг 2. Теперь мы хотим избавиться от сравнений, когда один из элементов, например $x$, принадлежит adom, а второй, например $u$, не принадлежит adom. Отметим, что равенства $x=u$ в этом случае ложны, поэтому можно ограничиться лишь рассмотрением неравенств. Введем временно два функциональных символа: верхнюю и нижнюю звездочки. Обозначим через $u^{*}$ ближайший к $u$ справа элемент множества adom, и если такого нет, то считаем $x^{*}=M$. Аналогично, $u_{*}$ - ближайший слева элемент множества adom или $u_{*}=m$, если такого нет. Теперь каждое неравенство $x<u$ эквивалентно $x<u^{*} \vee u_{*}=M$, а неравенство $x>u$ эквивалентно $x>u_{*} \vee u^{*}=m$.

Заметим, что для термов $u_{*}$ и $u^{*}$ не появятся формулы вида $E\left(u_{*}, v\right)$, $E\left(v, u_{*}\right), E\left(u^{*}, v\right)$ или $E\left(v, u^{*}\right)$.

Шаг 3. Сделаем следующее преобразование с целью исключить adom-ограниченные кванторы всюду, кроме автоматных подформул. Любой элемент $x \in$ adom можно идентифицировать с помощью такого элемента $\check{x}$ случайного графа, что для $\check{x}$ выполнены условие $(*)$ и формула $E(\check{x}, x)$. Поэтому каждую подформулу $(\exists x \in \operatorname{adom}) \phi$ можно заменить на $(\exists \check{x} \notin \operatorname{adom})\left(\Phi_{1}(\check{x}) \wedge \phi\right)$, преобразовав в $\phi$ атомные формулы, в которые входил элемент $x$, следующим образом:

1) $E(u, x)$ заменяем на $(\forall y \in \operatorname{adom})(E(\check{x}, y) \rightarrow E(u, y))$;

2) $E(x, u)$ заменяем на $(\forall y \in \operatorname{adom})(E(\check{x}, y) \rightarrow E(y, u))$;

3) $R(x)$ заменяем на $(\forall y \in \operatorname{adom})(E(\check{x}, y) \rightarrow R(y))$;

4) $x_{1}=x_{2}$ заменяем на $\Phi_{=}\left(\check{x}_{1}, \check{x}_{2}\right)$;

5) $x=M$ заменяем на $\Phi_{\max }(\check{x})$; 
6) $x=m$ заменяем на $\Phi_{\min }(\check{x})$;

7) $x_{1}<x_{2}$ заменяем на $\Phi_{<}\left(\check{x}_{1}, \check{x}_{2}\right)$.

Как легко видеть, все новые формулы являются автоматными. Ограниченный по adom квантор всеобщности преобразуется двойственным образом.

Точно таким же образом преобразуем термы вида $u_{*}$ и $u^{*}$, добавленные на шаге 2 , заменив их на термы $\check{u}_{*}$ и $\check{u}^{*}$ соответственно и преобразовав атомные формулы, в которые они входят, описанным выше способом.

Снова заметим, что для введенных переменных $\check{x}$ и термов $\check{u}_{*}, \check{u}^{*}$ не появятся ни сравнения, ни формулы вида $E(\check{x}, v)$ или $E(v, \check{x})$, кроме тех, которые входят в автоматные формулы.

Выполнив все перечисленные выше шаги 1-3, мы получим эквивалентную $\mu$ формулу вида

$$
\left(\mathrm{Q}_{1} v_{1}\right) \ldots\left(\mathrm{Q}_{k} v_{k}\right) \phi
$$

где $\phi$ является булевой комбинацией атомных и автоматных формул. При этом необходимо отметить, что в первый тип формул не будут входить переменные вида $\check{x}$ и термы вида $\check{u}_{*}, \check{u}^{*}$. Также напомним, что все переменные $v_{i}$ не входят в adom, поэтому для краткости мы не будем в кванторах выписывать $\notin$ adom. Предикаты $R \in \Omega$ будут встречаться только внутри автоматных подформул.

Далее индукцией по $l$ будем доказывать следующее

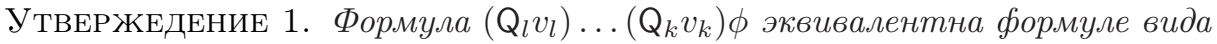

$$
\bigvee_{i}\left(\psi_{i} \wedge \theta_{i}\right)
$$

где $\theta_{i}$ - автоматные формуль, а формулы $\psi_{i}$ являются элементарными конбюнкииями, не содержащими переменных вида ц̌x и термов вида $\check{u}_{*}, \check{u}^{*}$.

ДоКАЗАТЕЛЬСтво. Базисом индукции будет случай $l=k+1$, т. е. случай, когда кванторы отсутствуют. Приведем формулу $\phi$ к дизъюнктивной нормальной форме; получим формулу вида

$$
\bigvee_{i}\left(\psi_{i} \wedge \bigwedge_{j} \eta_{i j}\right) .
$$

Здесь $\psi_{i}$ - конъюнкция атомных формул и их отрицаний, $\eta_{i j}$ - автоматные формулы и их отрицания. Согласно следствию 1 формула $\bigwedge_{j} \eta_{i j}$ снова эквивалентна некоторой автоматной формуле $\theta_{i}$, поэтому формула $\phi$ будет эквивалентна формуле

$$
\bigvee_{i}\left(\psi_{i} \wedge \theta_{i}\right)
$$

что и требовалось доказать.

Для обоснования индукционного шага отметим, что отрицание формулы вида (2) эквивалентно формуле такого же вида. Это следует из того, что отрицание автоматной формулы снова эквивалентно автоматной формуле. Получаем, что можно ограничиться рассмотрением только квантора существования, заменяя, когда необходимо, квантор всеобщноти квантором существования с отрицанием до и после него. 
Предположим, что для $l+1$ утверждение доказано, т. е. формула

$$
\left(\mathrm{Q}_{l+1} v_{l+1}\right) \ldots\left(\mathrm{Q}_{k} v_{k}\right) \phi
$$

эквивалентна формуле $\bigvee_{i}\left(\psi_{i} \wedge \theta_{i}\right)$. Рассмотрим формулу

$$
\left(\exists v_{l}\right)\left(\mathrm{Q}_{l+1} v_{l+1}\right) \ldots\left(\mathrm{Q}_{k} v_{k}\right) \phi \equiv\left(\exists v_{l}\right)\left(\bigvee_{i}\left(\psi_{i} \wedge \theta_{i}\right)\right) \equiv \bigvee_{i}\left(\exists v_{l}\right)\left(\psi_{i} \wedge \theta_{i}\right)
$$

Покажем, как преобразовать каждую формулу $\left(\exists v_{l}\right)(\psi \wedge \theta)$, чтобы снова получить формулу нужного вида.

Возможны два случая:

(i) переменная $v_{l}$ имеет вид $\check{x}$ и была введена на шаге 3 ;

(ii) переменная $v_{l}$ есть некоторая переменная $u$, которая была изначально ограничена по дополнению активной области.

Случай (i) достаточно тривиален, поскольку, как мы уже отмечали, переменная $\check{x}$ не может входить в $\psi_{i}$. Следовательно, получаем

$$
(\exists \check{x})\left(\psi_{i} \wedge \theta_{i}\right) \equiv \psi_{i} \wedge(\exists \check{x}) \theta_{i} .
$$

Согласно следствию 1 каждая формула $(\exists \check{x}) \theta_{i}$ эквивалентна некоторой автоматной формуле $\theta_{i}^{\prime}$, поэтому

$$
(\exists \check{x})\left(\mathrm{Q}_{l+1} v_{l+1}\right) \ldots\left(\mathrm{Q}_{k} v_{k}\right) \phi \equiv \bigvee_{i}\left(\psi_{i} \wedge \theta_{i}^{\prime}\right),
$$

что и требовалось показать.

Теперь рассмотрим случай (ii):

$$
(\exists u)(\psi \wedge \theta),
$$

причем для переменной $u$ внутри автоматной формулы $\theta$ могут встречаться термы типа $\check{u}_{*}$ и $\check{u}^{*}$, введенные на шаге 2 и модифицированные на шаге 3. Будем считать $\check{u}_{*}$ и $\check{u}^{*}$ внутри $\theta$ не сложными и зависящими от $u$ термами, а переменными; тогда формула (3) эквивалентна формуле

$$
\left(\exists \check{u}_{*}\right)\left(\exists \check{u}^{*}\right)(\exists u)\left(\psi \wedge \theta \wedge \Phi_{1}\left(\check{u}_{*}\right) \wedge \Phi_{1}\left(\check{u}^{*}\right) \wedge \chi\right),
$$

где $\chi$ описывает соотношения между $u, \check{u}_{*}$ и $\check{u}^{*}$, т. е. имеет следующий вид:

$$
\begin{aligned}
(\underbrace{\Phi_{=}\left(\check{u}_{*}, \check{u}^{*}\right) \wedge \Phi_{\min }\left(\check{u}^{*}\right) \wedge u<m}_{\chi_{1}}) & \vee(\underbrace{\Phi_{=}\left(\check{u}_{*}, \check{u}^{*}\right) \wedge \Phi_{\max }\left(\check{u}_{*}\right) \wedge u>M}_{\chi_{2}}) \\
& \vee(\underbrace{\Phi_{s}\left(\check{u}_{*}, \check{u}^{*}\right) \wedge u>^{*} \check{u}_{*} \wedge u<^{*} \check{u}^{*}}_{\chi_{3}}) .
\end{aligned}
$$

С помощью неравенств $u<^{*} \check{x}$ и $u>^{*} \check{x}$ мы обозначили утверждения о том, что $u$ меньше или больше соответственно единственного $y \in$ adom, для которого выполнено $E(\check{x}, y)$. 
Преобразуем формулу (4) к эквивалентной:

$$
\bigvee_{j=1}^{3}\left(\exists \check{u}_{*}\right)\left(\exists \check{u}^{*}\right)(\exists u)\left(\psi \wedge \theta \wedge \Phi_{1}\left(\check{u}_{*}\right) \wedge \Phi_{1}\left(\check{u}^{*}\right) \wedge \chi_{j}\right)
$$

Напомним, что $\theta$ - автоматная формула, а $\psi$ - элементарная конъюнкция. Если в $\psi$ есть равенство $u=v$, то автоматически получаем $u_{*}=v_{*}$ и $u^{*}=v^{*}$, следовательно, можно считать $\check{u}_{*}=\check{v}_{*}$ и $\check{u}^{*}=\check{v}^{*}$ и можно заменить переменные $u, \check{u}_{*}$ и $\check{u}^{*}$ на $v, \check{v}_{*}$ и $\check{v}^{*}$ соответственно.

Иначе формула $\psi$ в общем случае имеет вид

$$
\begin{aligned}
\psi^{\prime} & \wedge\left(\bigwedge_{i} u<v_{i}\right) \wedge\left(\bigwedge_{i} w_{i}<u\right) \wedge\left(\bigwedge_{i} E\left(u, a_{i}\right)\right) \\
& \wedge\left(\bigwedge_{i} \neg E\left(u, b_{i}\right)\right) \wedge\left(\bigwedge_{i} E\left(c_{i}, u\right)\right) \wedge\left(\bigwedge_{i} \neg E\left(d_{i}, u\right)\right) .
\end{aligned}
$$

Здесь переменные $a_{i}, b_{i}, c_{i}, d_{i}$ не имеют вид $\check{x}$, формула $\psi^{\prime}$ не содержит $u, \check{u}_{*}$ и $\check{u}^{*}$.

По очереди преобразуем части формулы (5) при $j=1,2,3$ к нужному нам виду.

При $j=1$ имеем формулу

$$
\left(\exists \check{u}_{*}\right)\left(\exists \check{u}^{*}\right)(\exists u)(\psi \wedge \underbrace{\theta \wedge \Phi_{1}\left(\check{u}_{*}\right) \wedge \Phi_{1}\left(\check{u}^{*}\right) \wedge \Phi_{=}\left(\check{u}_{*}, \check{u}^{*}\right) \wedge \Phi_{\min }\left(\check{u}^{*}\right)}_{\zeta} \wedge u<m) .
$$

Формула $\zeta$ является конъюнкцией автоматных формул, согласно следствию 1 она эквивалентна некоторой автоматной формуле $\theta^{\prime}$. Последнее неравенство можно включать в $\psi$, считая $m$ одним из $v$ в (6). Теперь согласно лемме 5 формула

$$
\left(\exists \check{u}_{*}\right)\left(\exists \check{u}^{*}\right)(\exists u)\left(\psi \wedge \theta^{\prime}\right)
$$

эквивалентна

$$
\psi^{\prime} \wedge\left(\bigwedge_{i, j} w_{i}<v_{j}\right) \wedge\left(\bigwedge_{i, j} a_{i} \neq b_{j}\right) \wedge\left(\bigwedge_{i, j} c_{i} \neq d_{j}\right) \wedge\left(\exists \check{u}_{*}\right)\left(\exists \check{u}^{*}\right)(\exists u) \theta^{\prime} .
$$

Формула $\left(\exists \check{u}_{*}\right)\left(\exists \check{u}^{*}\right)(\exists u) \theta^{\prime}$ согласно следствию 1 эквивалентна некоторой автоматной формуле $\theta^{\prime \prime}$. Остается только заменить неравенства вида $x \neq y$ на $x<y \vee x>y$.

При $j=2$ получаем формулу

$$
\left(\exists \check{u}_{*}\right)\left(\exists \check{u}^{*}\right)(\exists u)(\psi \wedge \underbrace{\theta \wedge \Phi_{1}\left(\check{u}_{*}\right) \wedge \Phi_{1}\left(\check{u}^{*}\right) \wedge \Phi_{=}\left(\check{u}_{*}, \check{u}^{*}\right) \wedge \Phi_{\max }\left(\check{u}_{*}\right)}_{\zeta} \wedge u>M),
$$

которая преобразуется аналогично случаю $j=1$ при одном исключении: в формулу (6) добавляем $M<u$, т. е. включаем $M$ как одно из $w$. 
Для $j=3$ будем иметь

$$
\left(\exists \check{u}_{*}\right)\left(\exists \check{u}^{*}\right)(\exists u)(\psi \wedge \underbrace{\theta \wedge \Phi_{1}\left(\check{u}_{*}\right) \wedge \Phi_{1}\left(\check{u}^{*}\right) \wedge \Phi_{s}\left(\check{u}_{*}, \check{u}^{*}\right)}_{\zeta} \wedge u>^{*} \check{u}_{*} \wedge u<^{*} \check{u}^{*}) .
$$

Строим автоматную формулу $\theta^{\prime}$, эквивалентную $\zeta$. Для совместности $u>^{*}$ $\check{u}_{*} \wedge u<^{*} \check{u}^{*}$ с неравенствами $u<v$ и $u>w$ из $\psi$ нужно, чтобы было $v>^{*} \check{u}_{*}$ и $w<^{*} \check{u}^{*}$. Последние две формулы эквивалентны $\Phi_{<}\left(\check{u}_{*}, \check{v}^{*}\right) \vee \Phi_{\max }\left(\check{v}_{*}\right)$ и $\Phi_{<}\left(\check{w}_{*}, \check{u}^{*}\right) \vee \Phi_{\min }\left(\check{w}^{*}\right)$ соответственно. Следовательно, формула

$$
\left(\exists \check{u}_{*}\right)\left(\exists \check{u}^{*}\right)(\exists u)\left(\psi \wedge \theta^{\prime} \wedge u>^{*} \check{u}_{*} \wedge u<^{*} \check{u}^{*}\right)
$$

будет эквивалентна

$$
\begin{aligned}
\psi^{\prime} & \wedge\left(\bigwedge_{i, j} w_{i}<v_{j}\right) \wedge\left(\bigwedge_{i, j} a_{i} \neq b_{j}\right) \wedge\left(\bigwedge_{i, j} c_{i} \neq d_{j}\right) \\
& \wedge\left(\bigwedge_{i}\left(\Phi_{<}\left(\check{u}_{*}, \check{v}_{i}^{*}\right) \vee \Phi_{\max }\left(\left(\check{v}_{i}\right)_{*}\right)\right)\right) \wedge\left(\bigwedge_{i}\left(\Phi_{<}\left(\left(\check{w}_{i}\right)_{*}, \check{u}^{*}\right) \vee \Phi_{\min }\left(\check{w}_{i}^{*}\right)\right)\right) \\
& \wedge\left(\exists \check{u}_{*}\right)\left(\exists \check{u}^{*}\right)(\exists u) \theta^{\prime} .
\end{aligned}
$$

Обозначим через $\xi$ вторую и третью строки последней формулы. Заметим, что формула $\xi$ построена из автоматных формул, поэтому согласно следствию 1 можно построить автоматную формулу $\theta^{\prime \prime}$, эквивалентную $\xi$. После этого остается только заменить неравенства вида $x \neq y$ порядковыми сравнениями. Утверждение доказано.

При $l=1$ получим, что формула $\left(\mathrm{Q}_{1} v_{1}\right) \ldots\left(\mathrm{Q}_{k} v_{k}\right) \phi$ эквивалентна булевой комбинации автоматных и базисных формул. Базисные формулы могут содержать только константы $m$ и $M$, для них истинность всех атомных формул заранее известна: $m=M$ и $m<M$ эквивалентны $\Phi_{e}$ и $\neg \Phi_{e}$ соответственно, остальные атомные формулы ложны. Таким образом, мы будем иметь булеву комбинацию автоматных формул, которая согласно следствию 1 снова будет эквивалентна некоторой автоматной формуле $\lambda$.

Согласно следствию 3 формула $\lambda$ является <-инвариантной, как и $\mu$, поэтому если их значения совпадают при каком-либо упорядочении случайного графа, то они эквивалентны для любого упорядочения. Теорема доказана.

СледСтвиЕ 4. Если замкнутая формула ф сигнатуры $(E,<, \Omega)$ является $<-$ <вариантной для линейно упорядоченных случайных графов, то задаваемый ею язык $L_{\phi}$ является автоматным.

СлеДСТВИЕ 5. <-инвариантное свойство $S$ монадических состояний над упорядоченным случайным графом может быть выражено формулой первого порядка тогда и только тогда, когда язык, задаваемый $S$, является автоматным. .

\section{§ 5. =-инвариантные формулы}

Теперь покажем, какие свойства состояния можно выразить с помощью =-инвариантных формул. 
ЛЕмма 6. Любая =-инвариантная в линейно упорядоченных случайных графах формула ф сигнатуры $(E,<, \Omega)$ выражает булеву комбинацию свойств следующего вида:

(**) мощность предиката $R_{i}$ равна одному из $N_{1}, \ldots, N_{m}$ или имеет вид $k j+l$ для некоторого натурального $j$, где $m, k, l, N_{1}, \ldots, N_{m}$ - натуральные константы.

ДокАЗАТЕЛЬСтво. Передвинем активную область состояния $\mathfrak{D}$ так, чтобы все элементы $R_{i}$ были бы меньше всех элементов $R_{j}$ при $i<j$. В силу $=$-инвариантности формулы ее значение не изменится.

Любая =-инвариантная формула является тем более <-инвариантной, следовательно, по теоремам 7, 6 истинность формулы $\phi$ на $(\mathfrak{G},<, \mathfrak{D})$ эквивалентна тому, что некоторый конечный автомат $\mathfrak{M}$ принимает слово $\mathfrak{d}$.

Далее доказываем лемму индукцией по количеству предикатных символов в сигнатуре $\Omega$.

Базисом индукции является случай, когда имеется один предикат $R_{1}$ (см. также $[18, \S 7.7])$. Тогда автомат $\mathfrak{M}$ имеет однобуквенный входной алфавит. Без ограничения общности считаем автомат $\mathfrak{M}$ детерминированным. Тогда его граф имеет один из двух видов:

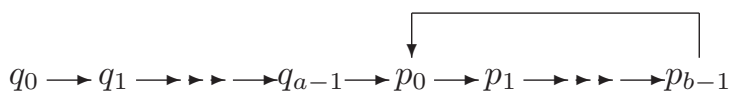

или

$$
q_{0} \longrightarrow q_{1} \longrightarrow \rightarrow q_{a-1}
$$

Пусть множество принимающих состояний автомата $\mathfrak{M}$ есть

$$
F=\left\{q_{i}: i \in F_{q}\right\} \cup\left\{p_{i}: i \in F_{p}\right\}
$$

Первый автомат принимает слова, содержащие $i \in F_{q}$ символов или $a+b j+i$ символов, $i \in F_{p}$, для любого натурального $j$. Второй автомат принимает слова, содержащие $i \in F_{q}$ символов.

Для обоснования индукционного шага предположим, что для $n$ предикатов теорема доказана. Рассмотрим произвольное состояние $\mathfrak{D}$ с $n+1$ предикатами.

Для каждого состояния $q$ автомата $\mathfrak{M}$ рассмотрим два автомата, получаемые из $\mathfrak{M}$ следующим преобразованием. Автомат $\mathfrak{M}_{q}^{\prime}$ получится, если из $\mathfrak{M}$ удалить все команды с символом $r_{n+1}$ и взять $\{q\}$ в качестве множества принимающих состояний. Для построения $\mathfrak{M}_{q}^{\prime \prime}$ удалим из $\mathfrak{M}$ все команды с символами $r_{i}, i \leqslant n$, и в качестве начального состояния возьмем $q$. Слово $\mathfrak{d}$ также разделим на два: префикс $\mathfrak{d}^{\prime}$, состоящий из символов $r_{i}, i \leqslant n$, и суффикс $\mathfrak{d}^{\prime \prime}$, состоящий только из $r_{n+1}$. Тогда легко видеть, что автомат $\mathfrak{M}$ принимает слово $\mathfrak{d}$ тогда и только тогда, когда для некоторого состояния $q$ автомат $\mathfrak{M}_{q}^{\prime}$ принимает слово $\mathfrak{d}^{\prime}$, a автомат $\mathfrak{M}_{q}^{\prime \prime}$ принимает слово $\mathfrak{d}^{\prime \prime}$. По индукционному предположению первое и второе может быть представлено булевой комбинацией свойств вида (**), обозначим эти комбинации через $S_{q}^{\prime}$ и $S_{q}^{\prime \prime}$ соответственно. Тогда автомат $\mathfrak{M}$ будет принимать слово $\mathfrak{d}$ в случае, когда $\mathfrak{D}$ обладает свойством $\bigvee_{q}\left(S_{q}^{\prime} \wedge S_{q}^{\prime \prime}\right)$. 
Теперь легко можем получить необходимый и достаточный критерий выразимости $=$-инвариантных в случайных графах свойств.

ТЕОРемА 8. =-инвариантное над линейно упорядоченными случайными графами свойство $\Omega$-состояний может быть выражено формулой тогда и только тогда, когда это свойство эквивалентно булевой комбинации свойств вида (**).

ДокАЗАТЕЛЬство. Необходимость следует из леммы 6.

Достаточность. Сначала нужно построить конечный автомат, проверяющий это свойство, а затем с помощью теоремы 5 построить автоматную формулу.

Построение автомата достаточно тривиально: сначала построим конечные автоматы $\mathfrak{M}_{i}$, проверяющие условия (**) для символа $r_{i}$, а затем - композицию автоматов $\mathfrak{M}_{i}$. Объединенный автомат будет принимать слово, если все автоматы к концу окажутся в принимающих состояниях.

\section{$\S 6$. Заключение}

Из полученных результатов можно извлечь некоторые результаты общего характера. Упорядочение случайного графа из теоремы 1, предложенное в [14], можно считать в некотором смысле самым простым, или наименее информативным, из всех возможных упорядочений, поскольку те свойства монадических состояний, которые описываются с его помощью, могут быть описаны и с помощью любого другого упорядочения.

С другой стороны, как мы уже отмечали (см. [10]), дискретное упорядочение случайного графа является неразрешимым и позволяет записывать любые арифметические свойства монадических состояний.

Нас интересует вопрос: существуют ли "промежуточные" варианты упорядочения случайного графа?

ВопРос 1. Существуют ли линейные упорядочения случайного графа с разрешимой теорией, которые позволяют выражать свойства монадических состояний, задающие неавтоматные языки?

Приведем более общий вопрос, ответ на который был бы крайне интересен.

Вопрос 2. Существуют ли универсумы с разрешимой теорией, которые позволяют выражать свойства монадических состояний, задающие неавтоматные языки?

\section{Список литературы}

1. E.F. Codd, "A relational model of data for large shared data banks", Comm. ACM, 13:6 (1970), 377-387.

2. E. F. Codd, "Relational completeness of data base sublanguages", Database systems, Prentice-Hall, San Jose, 1972, 33-64.

3. A. V. Aho, J. D. Ullman, "Universality of data retrieval languages", Conference Record of the Sixth Annual ACM Symposium on Principles of Programming Languages (San Antonio, TX, 1979), ACM, New York, 1979, 110-120. 
4. P. C. Kanellakis, G. M. Kuper, P. Z. Revesz, "Constraint query languages", J. Comput. System Sci., 51:1 (1995), 26-52.

5. С. М. Дудаков, М. А. Тайцлин, "Трансляционные результаты для языков запросов в теории баз данных", УМH, 61:2 (2006), 3-66; англ. пер.: S. M. Dudakov, M. A. Taitslin, "Collapse results for query languages in database theory", Russian Math. Surveys, 61:2 (2006), 195-253.

6. O. V. Belegradek, A. P. Stolboushkin, M. A. Taitslin, "Extended order-generic queries", Ann. Pure Appl. Logic, 97:1-3 (1999), 85-125.

7. J. Baldwin, M. Benedikt, "Stability theory, permutations of indiscernibles, and embedded finite models", Trans. Amer. Math. Soc., 352:11 (2000), 4937-4969.

8. С. М. Дудаков, "Трансляционный результат для расширений арифметики Пресбургера одноместной функцией, согласованной со сложением", Матем. заметки, 76:3 (2004), 362-371; англ. пер.: S. M. Dudakov, "Collapse result for extensions of the Presburger arithmetic by a unary function compatible with addition", Math. Notes, 76:3 (2004), 339-347.

9. С. М. Дудаков, "Трансляционная теорема для теорий $I$-сводимых алгебраических систем", Изв. РАН. Сер. матем., 68:5 (2004), 67-90; англ. пер.: S. M. Dudakov, "The collapse theorem for theories of I-reducible algebraic systems", Izv. Math., 68:5 (2004), 911-933.

10. M. Benedikt, L. Libkin, "Expressive power: the finite case", Constraint databases, Berlin, 1996, 55-87.

11. M. Benedikt, G. Dong, L. Libkin, L. Wong, "Relational expressive power of constraint query languages", J. ACM, 45:1 (1998), 1-34.

12. С. М. Дудаков, "Выразительная сила языков запросов первого порядка для баз данных на неупорядоченном случайном графе", Вестн. НовГУ, 34 (2005), 51-57.

13. M. Otto, J. Van den Bussche, "First-order queries on databases embedded in an infinite structure", Inform. Process. Lett., 60:1 (1996), 37-41.

14. С. М. Дудаков, "Разрешимая теория без трансляционной теоремы", Вестн. ТвГУ. Сер. Прикладная матем., 6:12 (2005), 23-26.

15. R. Rado, "Universal graphs and universal functions", Acta Arith., 9 (1964), 331-340.

16. A. K. Chandra, D. Harel, "Computable queries for relational data bases", J. Comput. System Sci., 21:2 (1980), 156-178.

17. Б. А. Трахтенброт, Я.М. Барздинь, Конечные автоматы (поведение и синтез), Наука, М., 1970; англ. пер.: В. А. Trakhtenbrot, Ya. M. Barzdin', Finite automata. Behavior and synthesis, North-Holland, Amsterdam-London, 1973.

18. В. Брауэр, Введение в теорию конечных автоматов, Радио и связь, М., 1987; пер. с нем.: W. Brauer, Automatentheorie, Leitfaden Monogr. Inform., Teubner, Stuttgart, 1984 .

C. М. Дудаков (S. M. Dudakov)

Тверской государственный университет

Поступило в редакцию

E-mail: Sergey.Dudakov@tversu.ru 15.01.2009 